\title{
EPP et bon usage des antibiotiques en réanimation : mobilisons-nous !
}

\author{
Professional practice evaluation and adequate use of antibiotics in the intensive care unit: we \\ have to mobilize!
}

\section{B. Schlemmer}

Reçu le 19 mars 2012 ; accepté le 20 mars 2012

(C) SRLF et Springer-Verlag France 2012

L'évolution préoccupante des résistances bactériennes aux antibiotiques rend indispensables de vigoureuses actions visant à maîtriser la transmission bactérienne dans les unités de soins, de même que l'usage des antibiotiques.

Dans un environnement microbien connu, responsable d'une certaine pression de colonisation par des espèces bactériennes résistantes ou susceptibles de le devenir par émergence de variants résistants, la pression de sélection exercée par les antibiotiques accroît en effet le risque d'émergence et de diffusion de la résistance. L'usage des antibiotiques est pourtant nécessaire à de nombreux malades pour qui le bénéfice de tels traitements est incontestable. C'est donc en termes de balance bénéfice/risque que doit être apprécié l'usage des antibiotiques et que la pratique professionnelle doit être abordée.

L'objectif de l'évaluation des pratiques professionnelles (EPP) est de confronter sa pratique à des standards dans le but de promouvoir la qualité des soins. La démarche est par essence pédagogique ; elle permet d'aller de l'évaluation de la pratique à l'évolution de la pratique. Encore faut-il avoir répertorié les points principaux qui assurent en général la pertinence d'un traitement antibiotique et garantissent la qualité de son suivi ; avoir considéré d'un œil critique les diverses recommandations de pratique expérimentées dans la littérature ; connaître celles de ces recommandations qui sont réputées avoir le plus fait évoluer la pratique et réduit l'usage inapproprié des antibiotiques.

Trois priorités apparaissent devoir être mises en exergue. Elles sont relatives aux procédures entourant la mise en route et le suivi de l'antibiothérapie.

La première apparaît devoir être d'ordre diagnostique. L'antibiothérapie de première intention est un exercice difficile. Il a maintenant été largement montré que le retard à la

\section{B. Schlemmer $(\bowtie)$}

Service de réanimation médicale, AP-HP, hôpital Saint-Louis ; Université Paris-Diderot, 1, avenue Claude Vellefaux, F-75010 Paris

e-mail : benoit.schlemmer@sls.aphp.fr mise en route d'un traitement approprié pesait sur le pronostic des malades. Si l'incertitude et l'urgence, devant un malade grave, peuvent légitimer des traitements relativement « larges », il est néanmoins indispensable d'assurer au préalable les conditions optimales du diagnostic (données cliniques, prélèvements microbiologiques, etc.) et de ne pas faire de ce traitement antibiotique initial une simple « couverture», en assurant les meilleures conditions de sa réévaluation.

La deuxième en est la suite logique. C'est en effet l'exigence de la réévaluation à 48-72 h du traitement initial, à confronter à l'état du malade et aux résultats des investigations microbiologiques préalablement conduites. Cette étape doit permettre de dire si le traitement initial est adapté, approprié, pertinent, en termes d'indication, de choix antibiotique, de posologies, de modalités d'administration, et enfin de sécurité pour le malade.

La troisième est relative à la traçabilité de l'antibiothérapie et des étapes qui ont fondé son initiation, sa poursuite ou son arrêt, de même que ses modalités. Elle relève donc de la qualité de la tenue du dossier du patient. La justification de chacune des décisions prises, le relevé des éléments cliniques qui les ont fondées, la relation des discussions tenues ou des choix opérés doivent pouvoir être retrouvés. Ils sont une marque de la qualité de la stratégie de prise en charge du malade et de la continuité des soins.

La promotion de l'EPPA (EPP en antibiothérapie) doit être assurée. Elle représente en effet un levier pour l'amélioration de l'usage des antibiotiques, et une action d'autoenseignement permanent.

Elle suppose cependant au préalable qu'une réflexion sur l'antibiothérapie ait été conduite au sein de l'unité par la totalité de l'équipe en charge des malades. Cette réflexion est l'affaire de tous, et pas seulement de tel ou tel membre de l'équipe qui se parerait des plumes de l'expert pour en imposer aux autres, à coup de recettes ou de préconisations standard. Si l'impact de l'intervention d'un spécialiste ou d'un « team antibiotique » sur la qualité de l'antibiothérapie a souvent été montré, il n'est pas certain que l'appropriation 
des règles de bonne pratique antibiotique ne soit pas mieux assurée à long terme par une démarche de type EPP, partagée par l'ensemble de l'équipe en charge des patients.

Construire une procédure d'EPPA en réanimation doit être une action collective, à inscrire dans l'ensemble de la démarche qualité d'un service. Celle-ci doit évidemment être adaptée aux caractéristiques de l'activité et à la typologie des patients pris en charge. La définition des grands axes d'une politique antibiotique au sein de l'unité est un préalable à la mise en route d'actions d'EPPA : elle doit porter sur la qualité de la démarche diagnostique, sur des recommandations consensuelles d'antibiothérapie dans un certain nombre de situations, sur des préconisations de suivi, de désescalade thérapeutique éventuelle, de durées de traitement, de précautions relatives aux posologies et modalités d'administration, etc.

L'action d'EPP peut ensuite être envisagée. Elle peut être soit d'ordre général, soit focalisée sur un aspect de l'usage des antibiotiques, comme par exemple les modalités d'utilisation de telle ou telle classe d'antibiotiques (fluoroquinolones), d'associations d'antibiotiques, etc. Des grilles d'éva- luation pour l'EPPA ont été proposées (Haute Autorité de santé, Société de pathologie infectieuse de langue française) et peuvent servir de base à la mise en route d'un tel programme. Il faut y encourager le plus grand nombre d'entre nous.

\section{Pour en savoir plus}

Dellit TH, Owens RC, Mc Gowan JE, et al (2007) Infectious diseases Society of America and the Society for Healthcare Epidemiology of America. Guidelines for Developing an Institutional Program to Enhance Antimicrobial Stewardship. Clin Infect Dis 44:59-77

Haute Autorité de santé (2008) Recommandations professionnelles Stratégie d'antibiothérapie et prévention des résistances bactériennes en établissement de santé. Grilles d'évaluation des pratiques professionnelles. www.has-sante.fr

Paterson RL (2006) The role of antimicrobial management programs in optimizing antibiotic prescribing within hospitals. Clin Infect Dis 42:S90-5

Société de pathologie infectieuse de langue française (2002) Comment améliorer la qualité de l'antibiothérapie dans les établissements de soins ? Med Mal Infect 32:320-8 